\title{
Optimization Design for Dynamic Characters of Electromagnetic Apparatus Based on Niche Sorting Multi-objective Particle Swarm Algorithm
}

\author{
Le Xu, Jiaxin You*, Haidan Yu, and Huimin Liang \\ School of Electrical Engineering and Automation, Harbin Institute of Technology, Harbin 150001, China
}

(Received 1 March 2016, Received in final form 27 August 2016, Accepted 29 August 2016)

\begin{abstract}
The electromagnetic apparatus plays an important role in high power electrical systems. It is of great importance to provide an effective approach for the optimization of the high power electromagnetic apparatus. However, premature convergence and few Pareto solution set of the optimization for electromagnetic apparatus always happen. This paper proposed a modified multi-objective particle swarm optimization algorithm based on the niche sorting strategy. Applying to the modified algorithm, this paper guarantee the better Pareto optimal front with an enhanced distribution. Aiming at shortcomings in the closing bounce and slow breaking velocity of electromagnetic apparatus, the multi-objective optimization model was established on the basis of the traditional optimization. Besides, by means of the improved multi-objective particle swarm optimization algorithm, this paper processed the model and obtained a series of optimized parameters (decision variables). Compared with other different classical algorithms, the modified algorithm has a satisfactory performance in the multi-objective optimization problems in the electromagnetic apparatus.
\end{abstract}

Keywords : electromagnetic apparatus, Multi-objective Particle Swarm Optimization (MOPSO), niche sorting strategy, dynamic characteristics

\section{Introduction}

With the rapid development of the weaponry such as the space station, the all-electric aircraft and the armored car, the requirement of the electrical systems is becoming higher and higher. The requirement specifically aims at the increment of power capacity, the improvement of reliability, the uninterrupted power supply and the automatic management of load. Hence the demand of high power electrical switch device is strongly promoted by the trend in higher electric power, and the research, experiment and production of switching device (including contactors) is facilitated by the trend [1-3]. However, the performance of the switching device, such as the voltage, the time, the closing velocity and the breaking velocity, is directly decided by the electrical and mechanical parameters. And the lifetime of the switching device is effected by the performance of the device. Therefore, it's highly important to provide an effective approach for the optimization of the high power electromagnetic apparatus.

(C)The Korean Magnetics Society. All rights reserved.

*Corresponding author: Tel: +86-0451-86413193

Fax: +86-0451-86413964, e-mail: ibic2013@126.com
The optimization of the electromagnetic apparatus dynamic characters is a multi-objective non-linear problem of the parameter, and the constraint is included in the optimization. Nowadays the related research at home and abroad mostly focused on the heuristic intelligent algorithms like the genetic algorithm. Nevertheless, most papers aimed at single target optimization without the consideration of several targets at the same time. The adjusting parameters of the electromagnetic apparatus were optimized by experimental design and analysis [4]. Studies were carried out on the optimization design of electromagnetic apparatus by classical algorithms such as artificial fishswarm algorithm and genetic algorithm [5-7]. However the foregoing researches were still integrated into a singleobjective problem, which couldn't be figured out simply by distributing some coefficients ignoring their own physical meanings.

Particle swarm optimization algorithm (PSO) has been applied into the nature science because of its rapid convergence and strong exploration, and also achieved rapid development in the multi-objective optimization problem in these years. However, the prematurity is resulted by rapid convergence inevitably. The improvement of the problem is urgently needed and lots of study works were 
carried out on it. Distance Sorting Multi-objective Particle Swarm Optimization (DSMOPSO) was built based on rank sort of crowding distance with the elitist strategy. What's more, a small ratio mutation was introduced to the inner swarm to enhance the global searching capacity of the algorithm and the number of Pareto optimal solutions could be controlled [8]. Angle Clustering Multi-objective Particle Swarm Optimization (ACMOPSO) algorithm was proposed by Kent in the design of aviation control system, and in the algorithm mapping and clustering analysis was applied [9]. To solve the electromagnetic optimization problem, modified Particle Swarm Optimization algorithm based on Gaussian distribution probability operator and considering mimicking territorial dispute between groups were applied respectively [10-12]. The general shortages still exist in kinds of multi-objective electromagnetic optimization problems such as the diversity and the distribution of the Pareto solutions.

Aiming at these problems, this paper designs an improved PSO algorithm with the niche sorting strategy, and the algorithm is successfully applied to the optimization of the high power plunger electromagnetic apparatus. The diversity and the distribution can be well guaranteed with the niche sorting, and the exploration ability can be enhanced simultaneously. Compared with traditional orthogonal experimental design and classical algorithm (NSGAII), the improved algorithm has a satisfactory performance in the multi-objective optimization problems of the electromagnetic apparatus.

\section{Multi-objective Particle Swarm Optimization algorithm based on the niche sorting}

\subsection{Standard Particle Swarm Optimization}

Particle Swarm Optimization algorithm (PSO) was proposed by psychologist Kennedy and electrical engineer Eberhart. PSO is a kind of swarm intelligence algorithm inspired by the birds interactive searching the best position of the group and individuals. PSO is described as follows.

$$
\begin{aligned}
v_{i, d}^{k+1}= & w \cdot v_{i, d}^{k}+c_{1} \cdot r_{i 1, d}^{k} \cdot\left(p_{i, d}^{k}-x_{i, d}^{k}\right) \\
& +c_{2} \cdot r_{i 2, d}^{k} \cdot\left(p_{g, d}^{k}-x_{i, d}^{k}\right) \\
x_{i, d}^{k+1}= & x_{i, d}^{k}+v_{i, d}^{k+1}
\end{aligned}
$$

The parameters $v_{i, d}^{k}$ and $x_{i, d}^{k}$ in (1) and (2) are the velocity and position of the particle $i$ at the iteration $k$ and dimension $d$, while $p_{i, d}^{k}$ and $p_{g, d}^{k}$ mean the individual historical best position and global best position. The variable $w$ is the inertia weight, which controls how the previous velocity affects the current one. A big coefficient helps to jump out of the local minimums, while a small coefficient is beneficial for the convergence and precision of the algorithm. Constants $c_{1}$ and $c_{2}$ are called learning factor, indicating how the global best position and individual historical best position influence on velocity. Constant $r$ is a random number at the interval $[0,1]$.

The PSO is called standard particle swarm optimization algorithm (SPSO) when the inertia weight $w$ is linearly decreasing.

\subsection{Niche Sorting Multi-objective Particle Swarm Optimization (NSMOPSO)}

The global best positions and individual historical best positions are the key factors for turning SPSO algorithm into MOPSO, whereas they are not unique any more for multi-objective problems. In this paper, the niche sorting multi-objective particle swarm optimization algorithm is introduced based on the MOPSO and the NSMOPSO structure as follows:

Step 1. Initiate particles swarm $N_{P}$; generate the initial position $X_{0}$ randomly and set the initial individual historical best position $P=X_{0}$; generate the initial velocity $V_{0}$ randomly; set the outer space $N_{s}$ as null.

Step 2. Evaluate the objective functions of every particle, and take the Pareto solutions into the outer space.

Step 3. Evaluate the fitness $F_{i}$ of per individual in outer space and rank the individuals by the decreasing order of the fitness, and the fitness is stated as (3); take the particle of the largest fitness as the global best position.

$$
F_{i}=\frac{1}{S_{i}}, i=1,2, \ldots, N_{S}
$$

where $N_{\mathrm{s}}$ is the number of individuals in the niche, and $S_{i}$ is the sharing degree of particle $i$, as shown in (4).

$$
S_{i}=\sum_{j=1}^{N_{S}} f_{s h}\left(d_{i j}\right), j=1,2, \ldots, N_{S}
$$

where $f_{s h}\left(d_{i j}\right)$ is the sharing function between particle $i$ and particle $j$, and it is defined by (5). The larger $f_{s h}\left(d_{i j}\right)$ is, the closer $X_{i}$ and $X_{j}$ is.

$$
f_{s h}\left(d_{i j}\right)=\left\{\begin{array}{l}
1-\left(\frac{d_{i j}}{\sigma_{\text {share }}}\right)^{\alpha}, 0 \leq d_{i j} \leq \sigma_{\text {share }} \\
0, d_{i j} \geq \sigma_{\text {share }}
\end{array}\right.
$$

where $d_{i j}$ stands for the distance between particle $i$ and particle $j, \alpha$ is the control parameter, and $\sigma_{\text {share }}$ is the predefined sharing radius.

Step 4. Update the position $x$ and velocity $v$ of particle $i$ by the equation (1) and (2); obtain new individual best 
position and the global best position. The inertia weight coefficient $w$ adopts the linear differential decreasing strategy as shown in (6) and (7).

$$
\begin{aligned}
& \frac{\mathrm{d} w(t)}{\mathrm{d} t}=\frac{2\left(w_{\text {start }}-w_{\text {end }}\right)}{t_{\text {max }}^{2}} \times t \\
& w(t)=w_{\text {start }}-\frac{\left(w_{\text {start }}-w_{\text {end }}\right)}{t_{\text {max }}^{2}} \times t^{2}
\end{aligned}
$$

Compared with the classical linear decreasing strategy taken by SPSO, the exploration of NSMOPSO at the early stage could be wider, it has faster convergence speed in the late period. Hence, the algorithm performance has been improved a lot.

Step 5. Update the outer space $N_{\mathrm{s}}$ by the Pareto solutions of current particles.

Step 6. Delete the particles of rather small fitness if the individual number exceeds limits.

Step 7. Judge whether it is terminate condition (algorithm converges or reaching maximum cycling times) or not. If not, go to Step 3, else output the Pareto solutions.

\section{The Optimization of the Electromagnetic Dynamic Characters Based on NSMOPSO}

3.1. The numeral solution of dynamic characters on the high power straightly-acting electromagnetic apparatus

The structure of the high power straightly-acting electromagnetic apparatus is shown in Fig. 1.

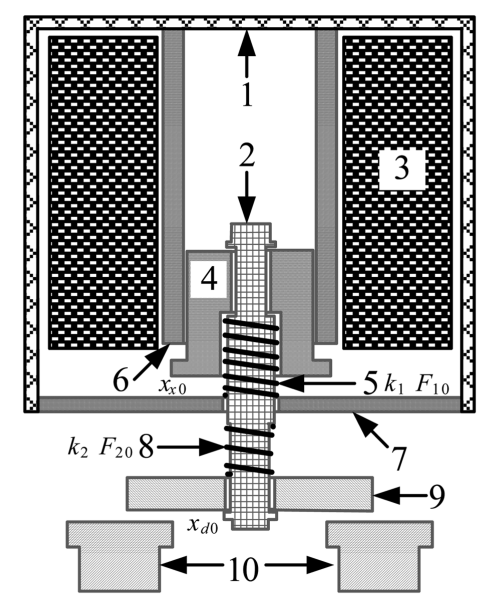

1-shell 2-link 3-coil 4-armature

5-reaction spring 6-iron core 7-yoke iron

8-bounce spring 9-travelling contact 10-fixed contact

$\mathrm{x}_{\mathrm{x} 0}$ - armature travel $\mathrm{x}_{\mathrm{d} 0}$-contact separation

$\mathrm{k}_{1}, \mathrm{k}_{2}, \mathrm{~F}_{10}, \mathrm{~F}_{20}$ - the stiffness and initial force of springs

Fig. 1. Structure of the high power straightly-acting electromagnetic apparatus.

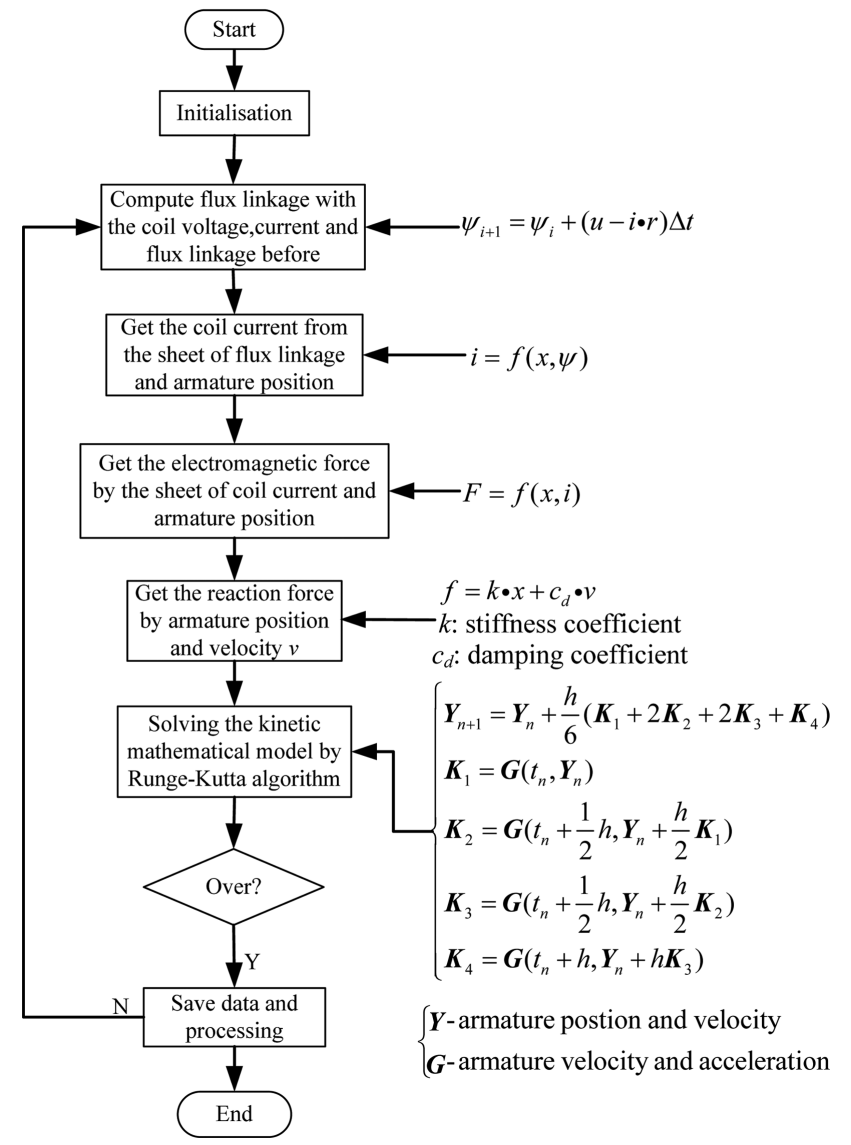

Fig. 2. Flowchart of the numeral solution of the dynamic characters.

The steps of the numeral solution are shown in Fig. 2. Firstly, set the parameters, such as the calculation step, coil drop time and collision parameter. Initialize parameters of the armature position, armature velocity, coil current, flux linkage and so on. Then, compute the flux linkage at the next moment with the voltage-equilibrium equation. Get the coil current from the sheet of flux linkage and armature position. Then get the electromagnetic force and reaction force separately. On this basis, solve the kinetic mathematical model by Runge-Kutta algorithm. Finally, save data and get the dynamic characters.

\subsection{Objective functions and optimal variables}

Considering the problems of closing bounce and weak breaking ability existed in the power straightly-acting electromagnetic apparatus, the contact closing velocity and the armature breaking velocity are proposed as the objective functions. The contact closing velocity influences the bounce strength while the armature breaking velocity decides the momentum of the rod and the breaking ability of the contacts. A small contact closing velocity is required to reduce the bounce while a large armature breaking 


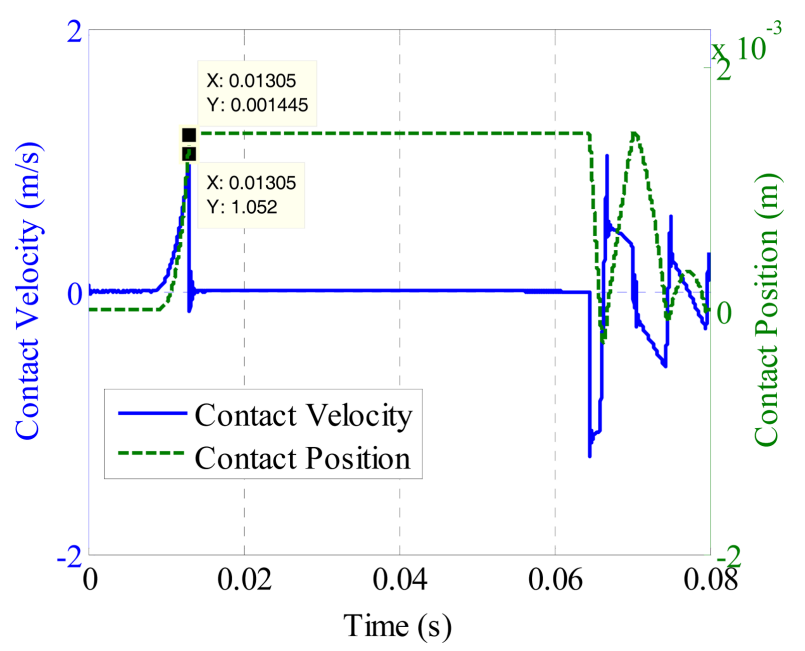

Fig. 3. (Color online) The contact position and velocity.

velocity is suggested to enhance the breaking ability. Therefore, the optimal design should be a multi-objective problem and the objective functions are the contact closing velocity and the armature breaking velocity.

(1) Contact closing velocity $v_{1}$. The contact velocity $v_{c}$ can be obtained by the numeral solution applied in the kinetic model of electromagnetic apparatus. The $v_{c}-t$ curve is shown in Fig. 3. The contact velocity increases to the largest when the contact position reaches the closing point, as depicted in (8).

$$
v_{1}=\max \left(v_{c}\right)
$$

(2) Armature breaking velocity $v_{2}$. The armature velocity $v_{a}$ can be obtained similar to the closing velocity. The $v_{a}-t$ curve is shown in Fig. 4. The armature velocity decreases to the minimum value when the contact breaks out, as

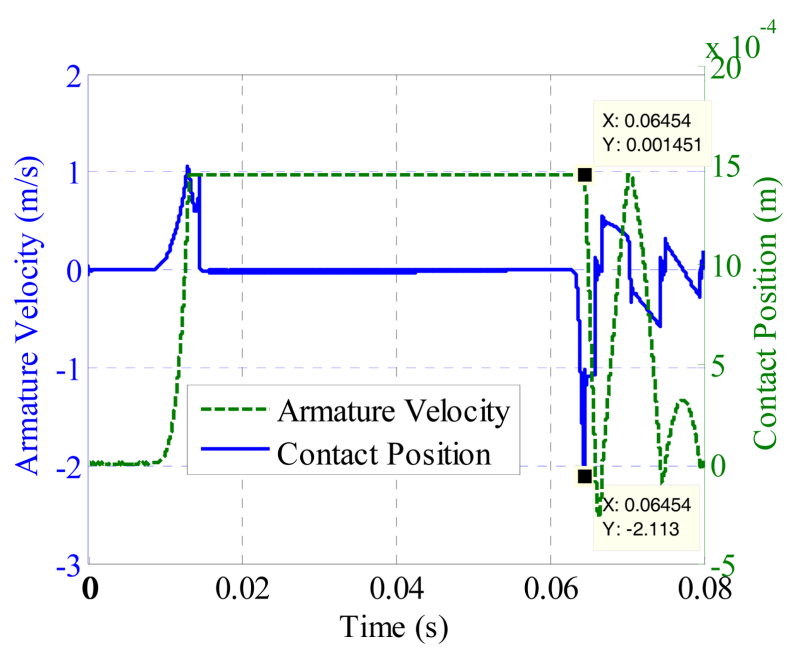

Fig. 4. (Color online) The armature velocity and contact position. depicted in (9).

$$
v_{2}=\min \left(v_{a}\right)
$$

In this case, the stiffness coefficients and initial forces of the two springs are selected as the optimal parameters $\left(k_{1}, k_{2}, F_{10}, F_{20}\right)$ because the reaction force is totally provided by the springs. Then, the optimal functions are defined as equation (10) and (11).

$$
\begin{aligned}
& f_{1}=\min \left(v_{1}\right) \\
& f_{2}=\min \left(v_{2}\right)
\end{aligned}
$$

\subsection{Constraint condition}

(1) Feasible region (boundary condition). Particles are limited to explore in the feasible region. The stiffness coefficient $(k)$ of the spring is mainly subjected to material and the manufacturing process $\left(k \in\left[k_{\min }, k_{\max }\right]\right)$. At the same time, the electromagnetic attraction force should cooperate well with the reaction force. Besides, the reaction force shouldn't be larger than the attraction force at pick-up voltage otherwise it will not be able to work properly. Therefore, the initial force of spring has its own domain $\left(F \in\left[F_{\min }, F_{\max }\right]\right)$.

(2) Other index constraints. Particles should be evaluated not only by the objective functions but also by the other index constraints. In this case, another target called pickup time is defined as the index constraint. The pick-up time indicates how long it takes for contact being closed totally after the coil is charged. The pick-up time is shown in Fig. 5, and it is limited in certain range by manufacturer $\left(t \leq t_{\max }\right)$.

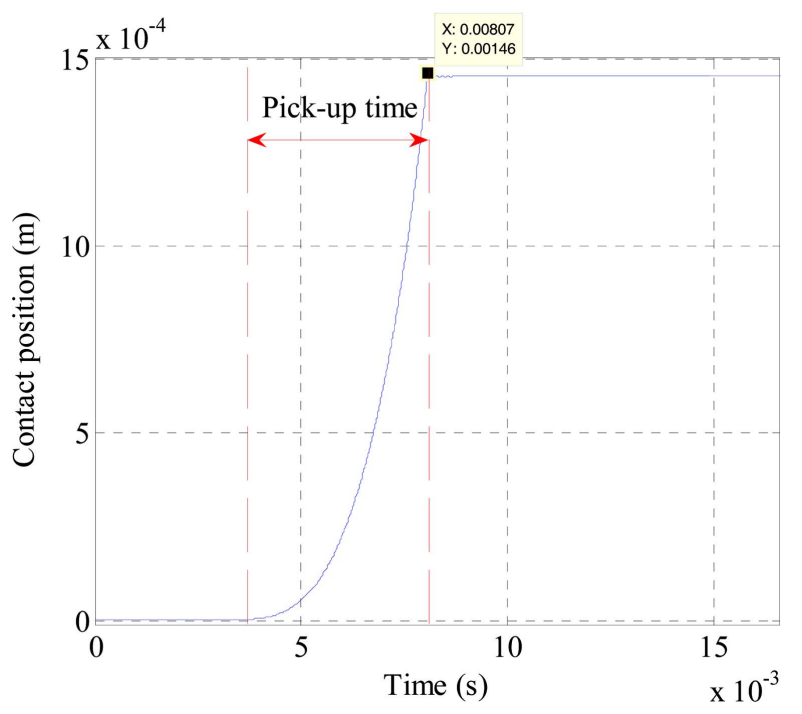

Fig. 5. (Color online) The sketch of pick-up time. 


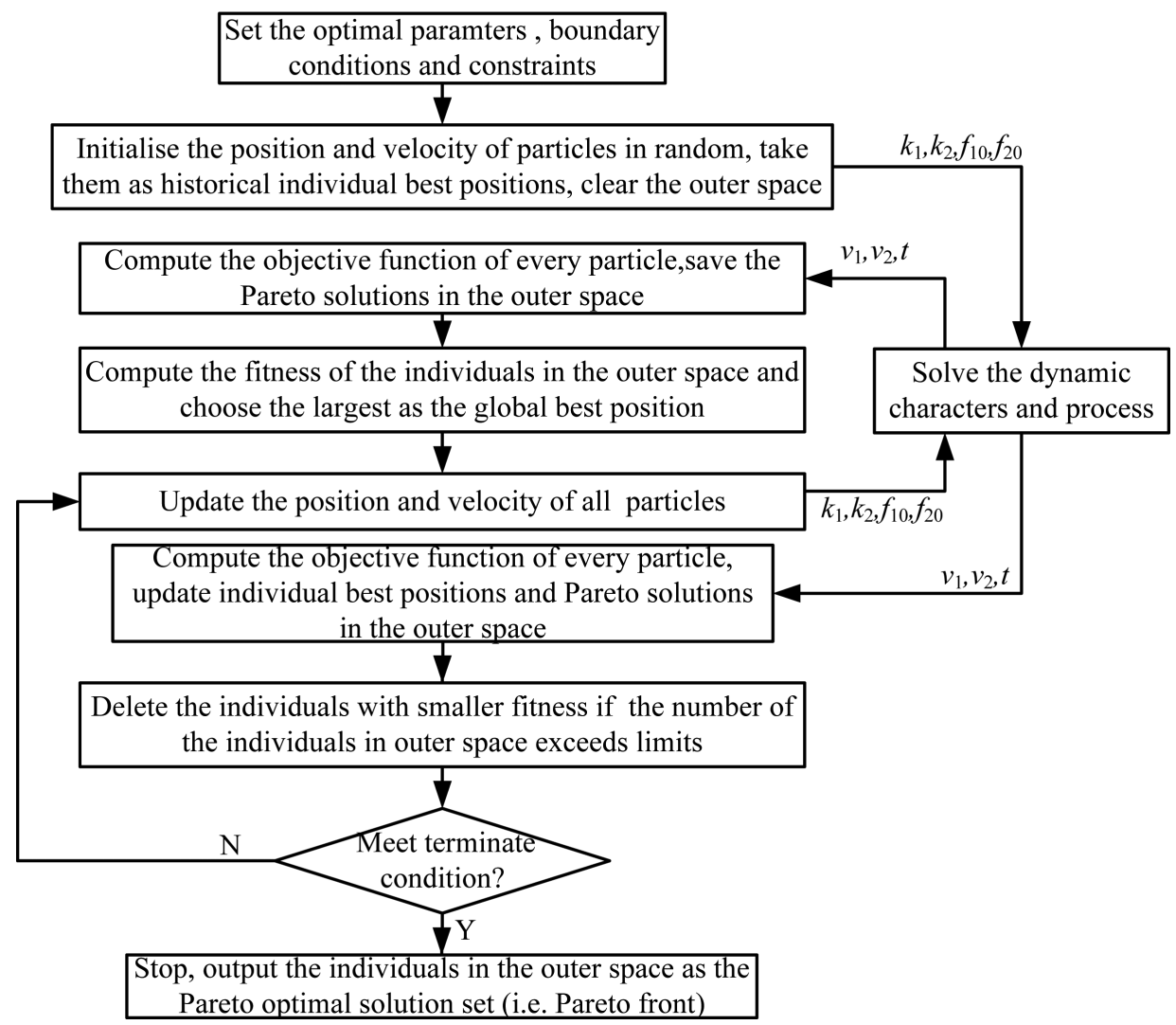

Fig. 6. Flowchart of the NSMOPSO algorithm interacting with the numeral solution module of dynamic characters.

\subsection{The multi-objective optimization process of dynamic characters of electromagnetic apparatus}

The multi-objective optimization of dynamic characters of electromagnetic apparatus is the interactive process. During the optimization, the NSMOPSO algorithm interacts with the numeral solution module of dynamic characters, aiming at searching for the best Pareto front. The flowchart is illustrated in Fig. 6.

\subsection{Experimental results and analysis}

In this section, the proposed NSMOPSO is compared with traditional orthogonal experimental design and classical genetic algorithm (NSGA-II) applied in the 400V/400A high power straightly-acting contactor. The boundary conditions and constraint are depicted in Table 1.

Considering the quite long time cost by the dynamic characters module, the number of particles and iterations

Table 1. Boundary conditions and index constraint.

\begin{tabular}{cccccc}
\hline \hline & $k_{1}(\mathrm{~N} / \mathrm{m})$ & $k_{2}(\mathrm{~N} / \mathrm{m})$ & $F_{10}(\mathrm{~N})$ & $F_{20}(\mathrm{~N})$ & $t(\mathrm{~s})$ \\
\hline $\min$ & 15000 & 300 & 1 & 20 & 0 \\
$\max$ & 20000 & 1000 & 10 & 5 & 0.02 \\
\hline
\end{tabular}

shouldn't be too large. Therefore, the number of individuals is set to 20 and the maximum number of iterations for all algorithms is 40. Other parameters of NSMOPSO algorithm are: the inertia weight coefficient $w_{\text {start }}=0.9$, $w_{\text {end }}=0.4$, the learning factor $c_{1}=c_{2}=2$, the predefined sharing radius $\sigma_{\text {share }}=1$, and the control parameter $\alpha=1$.

The results are presented in Fig. 7 and Table 2. Accord-

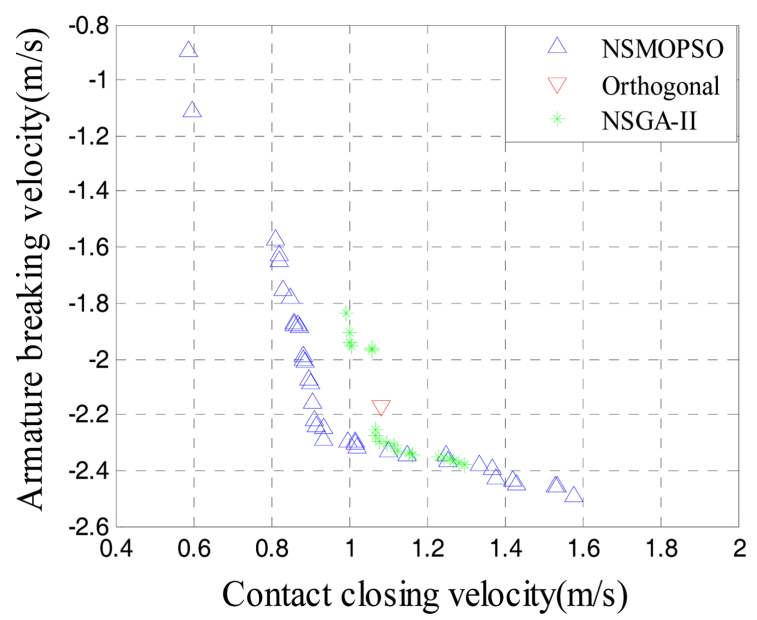

Fig. 7. (Color online) The Pareto front of different algorithms. 
Table 2. Comparison of different algorithms for the dynamic characters.

\begin{tabular}{ccccc}
\hline \hline & \multicolumn{2}{c}{ Extreme solution } & \multicolumn{2}{c}{ Compromise solution } \\
\cline { 2 - 5 } $\begin{array}{c}\text { Optimal } \\
\text { algorithm }\end{array}$ & $\begin{array}{c}\text { Closing } \\
\text { velocity } \\
\text { minimum } \\
(\mathrm{m} / \mathrm{s})\end{array}$ & $\begin{array}{c}\text { Breaking } \\
\text { velocity } \\
\text { minimum } \\
(\mathrm{m} / \mathrm{s})\end{array}$ & $\begin{array}{c}\text { Closing } \\
\text { velocity } \\
(\mathrm{m} / \mathrm{s})\end{array}$ & $\begin{array}{c}\text { Breaking } \\
\text { velocity } \\
(\mathrm{m} / \mathrm{s})\end{array}$ \\
\hline NSMOPSO & 0.5874 & -2.494 & 0.936 & -2.289 \\
Orthogonal & 1.02 & -2.317 & - & - \\
NSGA-II & 0.9906 & -2.383 & 1.071 & -2.275 \\
\hline
\end{tabular}

ing to the result, the proposed NSMOPSO algorithm has better exploration ability than orthogonal experimental design and classical genetic algorithm, which is inflected by the extreme solutions. What's more, the NSMOPSO algorithm has better distribution with a nice compromise solution.

Above all, the proposed NSMOPSO algorithm achieves quite a good performance in the solution of multiobjective optimization problem in the electromagnetic apparatus. It can effectively avoid premature convergence and converge rapidly. What's more, it can also make the distribution be wide and uniform and guarantee the diversity of the solutions. As a result, it can get the Pareto optimal solution set and keep the individual distribution uniform. Besides, the optimal solution can be chosen from the Pareto optimal solution set by practical preference or requirement for practical decision-making. It provides a reasonable way to optimize the electromagnetic apparatus instead of blindly dealing with weight percentages for multi-objective optimization problem.

\section{Conclusions}

In this paper, an improved multi-objective optimization algorithm named as niche sorting MOPSO has been introduced to solve the multi-objective optimization problem of the high power electromagnetic apparatus. The application analysis shows that NSMOPSO algorithm can avoid effectively premature convergence, realize convergence rapidly, and achieve wider and more uniform Pareto solution set than traditional orthogonal experimental design and classical genetic algorithm (NSGA-II). It provides a reasonable way to optimize the electromagnetic apparatus and decreases the blindness.

\section{Acknowledgments}

Authors express their thanks to the support of NSFC (National Natural Science Foundation of China) Project 51407041, 51507033 and China Postdoctor Science Foundation 2016M591530.

\section{References}

[1] R. K. Panda and Jitendar Veeramalla, Proc. IEEE 60th Int. Conf. Electrical Contacts 1 (2014).

[2] Ye Xuerong, Deng Jie, Wang Yingqi, and Zhai Guofu, J. Magn. 19, 378 (2014).

[3] Chen Degui, Liu Yingyi, Ji Liang, and Niu Chunping, IEICE Trans. Electron. E91-C, 8, 1280 (2008).

[4] Zhai Guofu and Zhou Xue, IEICE Trans. Electron. E92C, 1023 (2008).

[5] Chen Dewei and Zhang Peiming, Transactions of China Electrotechnical Society 26, 101 (2011).

[6] F. Mach, I. Novy, P. Karban, et al., ELEKTRO 2014, 596 (2014).

[7] S. Wang, Q. Li, and J. Chen, J. Magn. 19, 385 (2014).

[8] L. I. Zhong-Kai, J. R. Tan, Y. X. Feng, and H. Fang, Computer Integrated Manufacturing Systems 14, 1329 (2008).

[9] Liu Baoning, Zhang Weiguo, and Li Guangwen, Journal of Beijing University of Aeronautics and Astronautics 39, 458 (2013).

[10] Ren Ziyan, D. Um, and C. S. Koh, J. Magn. 19, 266 (2014).

[11] J. H. Seo, C. H. Im, S. Y. Kwak, C. G. Lee, and H. K. Jung, IEEE Trans. Magn. 44, 1046 (2008).

[12] Luiz Lebensztajn and Leandro dos Santos Coelho, IEEE 14th Int. Conf. Electromagnetic Field Computation. 1, (2010). 